\title{
Pediatric demyelinating disorders
}

\author{
Global updates, controversies, and future directions
}

Tanuja Chitnis, MD

Daniela Pohl, MD, PhD

On behalf of the

International Pediatric

MS Study Group

(IPMSSG) Steering

Committee

Correspondence to

Dr. Chitnis:

tchitnis@partners.org

Neurology ${ }^{\circledR}$ 2016;87 (Suppl 2):S1-S3
The field of pediatric-onset multiple sclerosis (MS) and other demyelinating diseases in childhood has advanced considerably since the publication of the last International Pediatric Multiple Sclerosis Study Group (IPMSSG)-sponsored Neurology ${ }^{\circledR}$ supplement in 2007. Over the last decade, demyelinating diseases of the CNS in children, and MS in particular, have garnered considerable international focus, both in the clinical and research world but also in the lay press and public awareness. This coming of age resulted in an exponential increase in research efforts and publications, with more than 250 peer-reviewed publications on pediatric MS in the past 10 years. It has also crystallized national and international collaborations that are critical to transform the field.

With an increase in diagnostic awareness of pediatric MS, the paradigm of early treatment emerged as a consensus standard of care. The rapidly expanding array of new immunomodulatory drugs approved for use in adult-onset MS has also resulted in the launch of the first pediatric MS clinical trials. These trials are pivotal to meaningfully steward powerful and efficacious therapies while addressing potentially more significant side effects and long-term risks. Identifying pediatric-specific considerations for safety and tolerability, defining outcome measures sensitive to MS expression in the pediatric context, and remaining vigilant for short- and long-term therapeutic impact on the developing nervous and immune systems in pediatric patients with MS is imperative. In addition to the need for clinical trials, pediatric MS care providers must also consider and contribute to evidence-based algorithms for diagnostic approaches and treatment management, including definitions for breakthrough disease. These guidelines have yet to be created but will be essential as the armamentarium of therapeutic options expands.

The current supplement summarizes new insights related to pediatric demyelinating disorders in 16 articles compiled under the direction of the IPMSSG Steering Committee, who identified topic experts. For each article, our aim was to encourage a team of international contributors with diverse areas of expertise to compile a critical state-of-the-art review on current knowledge, attain consensus on recommendations where clear-cut evidence is lacking, and discuss controversies and future directions. All first authors of the supplement were invited to review and provide final edits to all other articles, thereby further enhancing the consensus character of this supplement.

The first and most patient-centered article in this supplement is titled "Pediatric multiple sclerosis: Perspectives from adolescent patients and their families," depicting the day-to-day impact of a chronic neurologic disease at an early age. This article highlights the need for additional work on all areas to better treat and identify preventative and curative approaches for children and adolescents with MS. Further involvement of patients and their families in scientific efforts with the clinical and research community will help pave the way to a brighter future.

Major advances have been made in the diagnosis of pediatric MS, as summarized in "Consensus definitions for pediatric MS and other demyelinating disorders in childhood," showing that the McDonald 2010 criteria, as applied at the time of a first attack, have been validated in

From the Partners Pediatric MS Center (T.C.), Massachusetts General Hospital, Harvard Medical School, Boston; and Division of Neurology (D.P.), Children's Hospital of Eastern Ontario, University of Ottawa, Canada.

Go to Neurology.org for full disclosures. Funding information and disclosures deemed relevant by the authors, if any, are provided at the end of the article. 
patients 11 years and older and should be applied more cautiously in younger children. "Immunopathophysiology of pediatric CNS inflammatory demyelinating diseases" summarizes a deeper understanding of the pathophysiology of pediatric MS through the analysis of biopsy specimens, which demonstrate a $50 \%$ higher extent of acute axonal damage compared to adult patients with MS, particularly in the prepubertal age group. In addition, $\mathrm{T}$ cell studies reveal increased memory cells in pediatric patients with MS, and increased subsets of memory cells with a Th17 phenotype. "Environmental and genetic factors in pediatric inflammatory demyelinating diseases" summarizes results and ongoing efforts to identify genetic and environmental risk factors for pediatric MS, which may shed new light on the risk for MS overall. Present studies indicate commonality of genetic risk alleles including $H L A-D R B 1 * 1501$, and environmental risk factors including smoking exposure, hypovitaminosis D, obesity, and prior Epstein-Barr virus infection in children with MS, as previously observed in adult MS. The diagnosis of MS in childhood requires expertise in discerning MS from the broad spectrum of other immune and genetic disorders that can mimic MS in clinical expression and imaging characteristics. "Differential diagnosis and evaluation in pediatric inflammatory demyelinating disorders" provides guidance for clinicians with regard to the differential diagnosis of MS.

A pervasive theme among several articles is the identification of myelin-oligodendrocyte glycoprotein (MOG) antibodies in a variety of forms of demyelinating diseases in children. Although it is becoming clear that MOG antibodies are present in diverse pediatric demyelinating syndromes, and appear to predict a non-MS outcome in children with acute demyelinating syndromes, there is still much to be clarified about the clinical significance including diagnostic and prognostic values of these antibodies.

The current knowledge of the demyelinating syndromes optic neuritis $(\mathrm{ON})$, transverse myelitis (TM), and acute disseminated encephalomyelitis (ADEM) are summarized in "Acute disseminated encephalomyelitis: Updates on an inflammatory CNS syndrome,"
"Pediatric transverse myelitis," and "Pediatric optic neuritis." A major advance is the use of optical coherence tomography in pediatric patients, which demonstrates pathology in non$\mathrm{ON}$ eyes even in the early stages of the disease, signifying early silent tissue injury. ADEM is possibly not a single specific entity, but an inflammatory CNS syndrome with immunemediated demyelination and strong predilection to young children. TM is a potentially devastating condition with significant cumulative demands on health and social care resources, and ongoing trials will hopefully inform on optimal treatment of TM. Recently, there has been increased recognition that neuromyelitis optica spectrum disorders occur in children, as summarized in "Neuromyelitis optica spectrum disorders in children and adolescents." Further efforts are underway to evaluate the frequency and clinical associations of aquaporin- 4 and MOG antibodies in this cohort. "Pediatric acquired CNS demyelinating syndromes: Features associated with multiple sclerosis" highlights clinical and MRI predictors of development of MS after an acute demyelinating syndrome. Common risk factors are age, female sex, presence of brain MRI lesions, and specific lesion features.

"Pediatric multiple sclerosis: Clinical features and outcome" and "Pediatric multiple sclerosis: Cognition and mood" summarize the clinical and cognitive features of pediatric MS. An emerging theme is the difference in clinical and MRI presentation of prepubertal and postpubertal onset MS, which raises questions about the modifying effect of puberty. "MRI in the evaluation of pediatric multiple sclerosis" discusses the role of MRI in the evaluation of pediatric MS. Studies of volumetric MRI analysis demonstrate an impact of MS on age-expected brain growth and functional MRI reveals activation patterns in pediatric patients with MS that differ from regional brain activation patterns seen in healthy agematched youth. These studies highlight that MS onset during childhood may profoundly influence maturing and actively myelinating neural networks.

"Pediatric multiple sclerosis: Conventional first-line treatment and general management" summarizes the current knowledge of safety 
and efficacy of traditional first-line injectable agents, $\beta$-interferon and glatiramer acetate, which have been shown to be fairly safe in children with MS, although there are limited efficacy data. There have been major advances in the area of MS therapeutics, in which formal evaluation through randomized clinical trials of new MS therapies including oral drugs are now being conducted in children, as summarized in "Pediatric multiple sclerosis: Escalation and emerging treatments." Such trials have the potential to greatly advance the knowledge of safety and efficacy of newer agents, but also create new challenges for the pediatric MS community to evaluate the growing number of MS therapies.

"International Pediatric MS Study Group Global Members Symposium report" is a meeting report of the first global IPMSSG meeting held in Boston in 2014, during which major themes highlighted above were discussed. In addition, focused workshops were held to discuss vitamin $\mathrm{D}$ dosing in pediatric MS, cognition and its effects on real-world outcomes in childhood MS, and MS treatment approaches.

Pediatric MS and other inflammatory CNS demyelinating diseases constitute a group of disorders with potentially devastating irreversible neurologic consequences. We hope that this supplement will facilitate the best possible care for those young patients, by providing the knowledge basis and expert opinions required for informed decisions with regard to diagnostic and treatment strategies. Future research will hopefully elucidate preventative approaches, and provide more reliable information with regard to age-specific disease immunopathophysiology, as well as treatment risks and responses. In view of the relatively low incidence of pediatric demyelinating disorders, a collaborative, international approach is required. The IPMSSG will continue to support such international efforts and we strongly encourage all interested colleagues involved in the field of pediatric demyelinating disorders to actively participate in our projects (www. ipmssg.org).

This supplement is made possible by the financial support of MS organizations that in scope reflect the international collaboration in the field of pediatric MS. You will find the sponsors identified by name on the title page. We greatly appreciate their support, and thank their many donors worldwide.

\section{ACKNOWLEDGMENT}

Contributors: IPMSSG Steering Committee Members: Maria Pia Amato: Department NEUROFARBA, Section Neurosciences, University of Florence, Italy; Brenda Banwell: The Children's Hospital of Philadelphia, Perelman School of Medicine, University of Pennsylvania, Philadelphia; Angelo Ghezzi, Divisione di Neurologia 2-Centro Studi Sclerosi Multipla, Ospedale di Gallarate, Gallarate, Italy; Rogier Q. Hintzen: Department of Neurology, MS Centre ErasMS, Neurology, Erasmus MC, Rotterdam, the Netherlands; Lauren B. Krupp: Lourie Center for Pediatric MS, Stony Brook Children's Hospital, Stony Brook University, New York; Kevin Rostásy: Department of Pediatric Neurology, Children's Hospital Datteln, University Witten/Herdecke, Germany; Silvia Tenembaum: Department of Neurology, National Pediatric Hospital Dr. Juan P. Garrahan. Ciudad de Buenos Aires, Argentina; Evangeline Wassmer: Department of Neurology, Birmingham Children's Hospital, UK; Emmanuelle Waubant: Pediatric MS Center, UCSF Benioff Children's Hospital, and Neurology Department, UCSF, San Francisco, CA. IPMSSG Coordinating Consultant: Jon Temme.

\section{STUDY FUNDING}

This supplement is made possible by funding from the MS Cure Fund, Danish MS Society, German MS Society, Italian MS Association, MS International Federation, MS Research Foundation (Netherlands), National MS Society (USA) and Swiss MS Society.

\section{DISCLOSURE}

T. Chitnis has received personal compensation for advisory board/consulting from Biogen-Idec, Merck-Serono, and Novartis, and has received research support from Merck-Serono and Novartis Pharmaceuticals. She serves on pediatric clinical trial advisory boards for GenzymeSanofi and Novartis. D. Pohl has received compensation for consulting or conference presentations from Bayer, Biogen-Idec, Merck-Serono, Novartis, Sanofi, and Teva. Go to Neurology.org for full disclosures.

Received August 20, 2015. Accepted in final form March 16, 2016. 


\section{Neurology}

Pediatric demyelinating disorders: Global updates, controversies, and future directions Tanuja Chitnis and Daniela Pohl Neurology 2016;87;S1-S3

DOI 10.1212/WNL.0000000000002882

This information is current as of August 29, 2016

Updated Information \& Services

Subspecialty Collections

Permissions \& Licensing

Reprints including high resolution figures, can be found at: http://n.neurology.org/content/87/9_Supplement_2/S1.full

This article, along with others on similar topics, appears in the following collection(s):

Acute disseminated encephalomyelitis

http://n.neurology.org/cgi/collection/acute_disseminated_encephalomy elitis

All Demyelinating disease (CNS)

http://n.neurology.org/cgi/collection/all_demyelinating_disease_cns All Pediatric

http://n.neurology.org/cgi/collection/all_pediatric

Multiple sclerosis

http://n.neurology.org/cgi/collection/multiple_sclerosis

Information about reproducing this article in parts (figures,tables) or in its entirety can be found online at:

http://www.neurology.org/about/about_the_journal\#permissions

Information about ordering reprints can be found online:

http://n.neurology.org/subscribers/advertise

Neurology ${ }^{\circledR}$ is the official journal of the American Academy of Neurology. Published continuously since 1951, it is now a weekly with 48 issues per year. Copyright () 2016 American Academy of Neurology. All rights reserved. Print ISSN: 0028-3878. Online ISSN: 1526-632X.

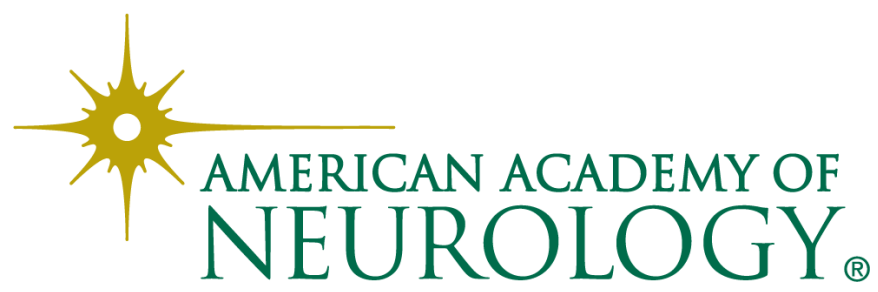

